\title{
Achievements of the First Generation of Monolithic Active Pixel Sensors for Charged Particle Tracking
}

\author{
Marc Winter* and Grezgorz Deptuch, Yuri Gornushkin ${ }^{\dagger}$ \\ IReS, IN2P3-CNRS/ULP, BP 28, 67037 Strasbourg, France \\ E-mail: 'marc. winterdires.in2p3. fir
}

\section{Gilles Claus, Wojciech Dulinski}

LEPSI, IN2P3/ULP, 23 rue du loess, BP 20, 67037 Strasbourg cedex 02, France

\begin{abstract}
The increasing need of high performance flavour tagging capabilities in particle physics experiments has triggered the development of a novel - fully integrated - silicon pixel detector, called Monolithic Active Pixel Sensor (i.e. MAPS). Similar to CMOS visible light imagers, the first MAPS prototypes adapted to the detection of minimum ionising particles (i.e. mip) were designed and fabricated in standard CMOS technology. Their tests with relativistic charged particles demonstrate that the sensors detect mips with very high efficiency and signal-to-noise ratio and provide excellent spatial resolution. The main aspects of these results, which establish the adequacy of the technology for charged particle tracking, are summarised in this paper, together with preliminary results on its radiation hardness. An outlook on the R\&D started to provide fully integrated building blocks of future vertex detectors is also provided.
\end{abstract}

\section{Introduction}

Vertex detectors are of growing importance in particle physics experiments as the knowledge of the event flavour is becoming an issue for several aspects of the most fundamental questions to investigate in the coming decades. Existing technologies (i.e. $\mathrm{CCDs}^{1}$ and hybrid pixels) exhibit high performances but those needed to take full advantage of future experimental conditions may be too demanding, though substantial improvements are still expected from both technologies. Originally developed for visible light imaging, a domain

\footnotetext{
${ }^{*}$ Speaker.

${ }^{\dagger}$ visitor from the Joint Institute of Nuclear Research, Dubna, Russian Federation.

${ }^{1}$ i.e. Charged Coupled Devices
} 
where they have proven to be very successful, CMOS sensors may have the intrinsic potential of merging the specific advantages of both technologies: they provide a high spatial resolution and can be thinned down like CCDs, and are likely to be much faster as well as much more resistant to neutron radiation than CCDs.

\section{Principle of operation}

The principle of charged particle detection with a MAPS is based on a concept similar to the one proposed in [i] $\left[\begin{array}{l}1 \\ 1\end{array}\right]$ for light imaging, where a thin epitaxial layer of low-resistivity silicon is used as a sensitive detector volume. The charge generated by the impinging particle at a rate of about 80 electron-hole pairs per micrometer is collected by a n-well/p-epi diode, created by n-well implantation into the epitaxial layer. The electrons liberated in this layer diffuse towards the diode within a typical time of a few tens of nanoseconds. Because of the three orders of magnitude between the doping levels of the p-epitaxial layer and of the neighbouring $\mathrm{p}^{++}$wells and substrate, potential barriers are created at the region boundaries, that act like mirrors for the excess electrons (Fig.1). The detection of charged particles with the principle exposed above was experienced with two prototype chips made of pixel matrices of slightly different design and tested with high energy particle beams in 1999-2000. The design principle and the main test results are reviewed hereafter.

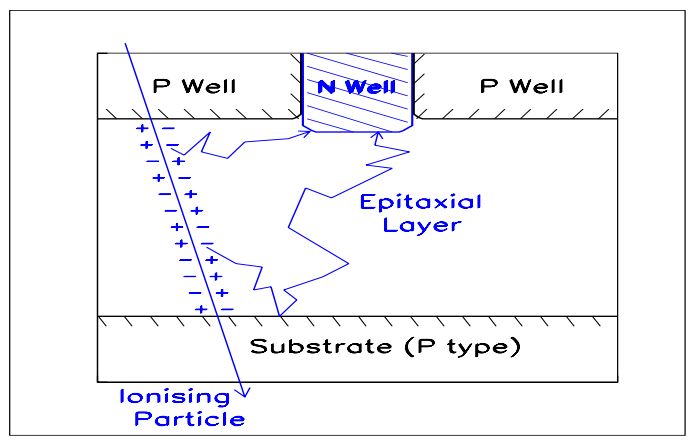

Figure 1: Schematic view of the internal structure of a pixel designed for charged particle tracking.

\section{Detector architecture}

The two prototypes, called MIMOSA${ }^{2}$, were fabricated in two different CMOS processes. MIMOSA-1 was fabricated in a 0.6- $\mu \mathrm{m}$ process featuring an epitaxial layer of about $14 \mu \mathrm{m}$, whereas MIMOSA-2 was fabricated in a $0.35-\mu m$ process with less than $5 \mu m$ epitaxial layer thickness. MIMOSA-1 (resp. MIMOSA-2) contain four (resp. six) independent matrices of active elements having slightly different designs. Each matrix consists of $64 x 64,20 \mu \mathrm{m}$ wide, pixels. The individual pixel is comprised of only three MOS transistors and a floating diffusion diode. The diode was implemented at the center of each pixel in most matrices. For one MIMOSA-1 matrix, each pixel hosted four diodes connected in parallel, and one MIMOSA-2 matrix hosted two diodes per pixel. This was supposed to reduce the charge dispersion and collection time, at the expense of increased noise reflecting the higher node capacitance and the smaller charge-to-voltage conversion gain. Compared to MIMOSA-1,

\footnotetext{
${ }^{2}$ standing for Minimum Ionising MOS Active pixel sensor.
} 
the design of MIMOSA-2 incorporates new ideas, including features improving the read-out speed and the radiation hardness.

The much thinner epitaxial layer of MIMOSA-2 was expected to translate into less signal than MIMOSA-1. This feature was compensated by a lower noise level consecutive to smaller node capacitance and very small diode leakage current. Basic prototype parameters, such as the total conversion gain and the pixel Equivalent Noise Charge (ENC), were determined with a $5.9 \mathrm{keV}$ X-ray source of ${ }^{55} \mathrm{Fe}$. The charge collection time of MIMOSA-1 was measured with laser shots to be less than $150 \mathrm{~ns}$. As a consequence of the thinner epitaxial layer, it is expected to be less than $100 \mathrm{~ns}$ for MIMOSA-2. More details on the chip architecture, principle of operation, results of the device simulation and of the tests with the X-ray source can be found in [i2i], [i3i].

\section{Tests with high momentum charged particles}

Both MIMOSA chips were tested with a pion beam of $120 \mathrm{GeV} / \mathrm{c}$ at the CERN SPS. A beam telescope of 8 planes of high precision silicon strip detectors, grouped in pairs of planes providing two orthogonal coordinates, was used to define the beam particle trajectory and intersection point with the MIMOSA plane. A pair of small scintillator counters provided the trigger for the data acquisition system, based on a VME-OS9 processor. Tracks were reconstructed by adjusting a straight line to the 8 telescope coordinates. The intersection of the tracks with the sensor plane was known within $\sim 1 \mu \mathrm{m}$. The off-line signal processing started with Correlated Double Sampling (CDS) [4] to eliminate some dominant noise components. The remaining noise was evaluated from the first 250 events of every run and used to compute the pedestals subtracted from the CDS outcome as well as the noise entering the signal-to-noise $(\mathrm{S} / \mathrm{N})$ calculation. A cluster finding algorithm, using pixels with $\mathrm{S} / \mathrm{N}>5$ as a seed, reconstructed clusters matching the beam telescope prediction.

The charge collected in the cluster is displayed on Fig.2 (left) as a function of the cluster multiplicity. The values found are in agreement with the simulation results 阿. As predicted, the 4 diodes/pixel option of MIMOSA-1 provided the most concentrated charge distribution. The charge collected with MIMOSA-2 (1 diode/pixel) was less spread than with MIMOSA-1, as expected from the thinner epitaxial layer.

The coordinate of the track impact was measured using the charge distribution in the cluster. After a precise alignment of the prototypes w.r.t. the telescope, the width of the distribution of the residue between the telescope extrapolation and the impact position provided by the sensors varied from 1.7 to $2.5 \mu \mathrm{m}$, depending on the matrix tested (see Fig. 2 - right). The best resolution was achieved with MIMOSA-1, with 1 diode/pixel, which exhibited the widest charge distribution and the highest $\mathrm{S} / \mathrm{N}$ ratio. After subtraction of the track prediction resolution $(\sim 1 \mu \mathrm{m})$, the intrinsic resolution of MIMOSA-1 was better

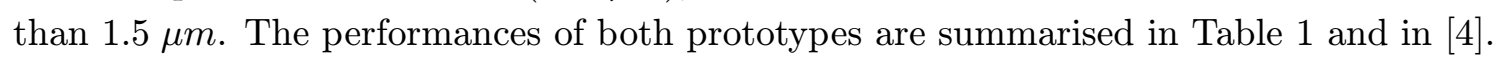



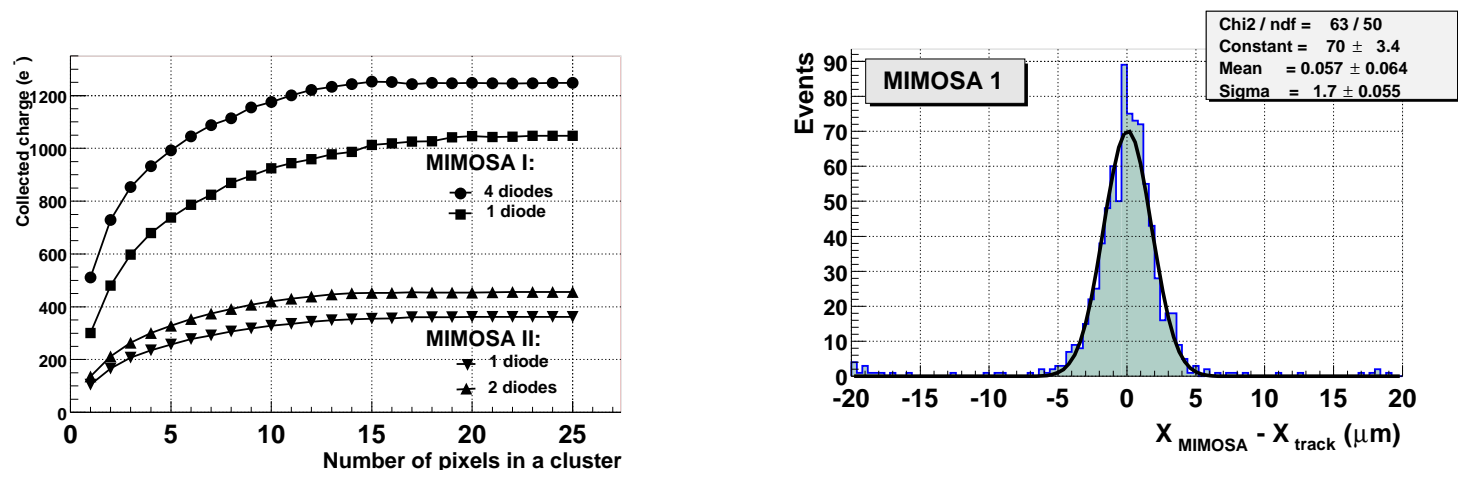

Figure 2: Left: Collected charge (most probable value) as a function of the cluster multiplicity for MIMOSA-1 and -2. Right: Residue distribution between the track position reconstructed with the MIMOSA-1 sensor (1 diode/pixel) and the position provided by the telescope.

\begin{tabular}{|c|c|c|c|}
\hline Prototype & \multicolumn{2}{|c|}{ MIMOSA-1 } & MIMOSA-2 \\
\hline Number of diodes/pixel & 1 & 4 & 1 \\
\hline Pixel noise (mean value) [e ${ }^{-}$ENC] & 12 & 25 & 9 \\
Signal-to-noise ratio (mean value) & 42 & 32 & 22 \\
Detection efficiency [\%] & $99.5 \pm 0.2$ & $99.2 \pm 0.2$ & $98.5 \pm 0.3$ \\
Spatial resolution [ $\mu \mathrm{m}]$ & $1.4 \pm 0.1$ & $2.1 \pm 0.1$ & $2.2 \pm 0.1$ \\
\hline
\end{tabular}

Table 1: Major performances of MIMOSA-1 (1 and 4 diodes per pixel) and -2 (1 diode per pixel). The noise is shown after off-line CDS and pedestral subtraction.

\section{Preliminary results on radiation tolerance}

The two prototypes were irradiated with $30 \mathrm{MeV} / \mathrm{c}$ protons and $10 \mathrm{keV} \mathrm{X}$-rays. Irradiation effects were investigated by studying changes in the sensor response to $5.9 \mathrm{keV}$ photons emitted by a ${ }^{55} \mathrm{Fe}$ source. It was observed that $600 \mathrm{kRad} \mathrm{X}$-rays provoke an increase of the diode leakage current by more than an order of magnitude. Similarly, a dose of $5.11^{10}$ $\mathrm{p} / \mathrm{cm}^{2}$ induced a factor of 5 increase of the diode leakage current and a loss in the charge collected of about $40 \%$.

On-going studies of sensors exposed to neutrons sources will clarify in which extent the observed sensitivity to protons is due to mechanical deformations of the epitaxy or if it originates from other degraded parts of the sensors (e.g. interfaces). Independently of the outcome of this study, present results on the radiation tolerance of CMOS sensors are encouraging, the first MIMOSA prototypes satisfying already the requirements of several applications (including those of a future $e^{+} e^{-}$linear collider). Moreover, the influence of the temperature on the chip performances will be investigated in order to reduce its sensitivity and to recover (at least part of) the observed performance losses. Finally, forthcoming sensor prototypes will allow to continue exploring the radiation tolerance of the sensors and to design chips exploiting more and more efficiently the real potential of their technology. 


\section{Outlook: Next R\&D steps}

The validity of the CMOS sensor detection principle for charged particle being established, the present phase of development aims to explore the technology potential of the sensors (by testing alternative sensing devices, studying and improving the sensor radiation tolerance, etc.) and to adapt them to various applications. Recently, a third prototype was fabricated in a standard $0.25 \mu \mathrm{m}$ deep submicron IBM process with $8 \times 8 \mu m^{2}$ wide pixels, and a fourth chip was manufactured in a $0.35 \mu \mathrm{m}$ AMS process with no epitaxy. These new arrays, which host various sensing devices, are currently being tested.

Aiming to validate the sensors as building blocks of future vertex detectors, the design of the first real scale prototype (MIMOSA-5) was recently sent for fabrication in the same $0.6 \mu \mathrm{m}$ process as MIMOSA-1. The chips have the size of a CMOS reticle (i.e. about $19.4 \times 17.4 \mathrm{~mm}^{2}$ ) and consist of 4 independent matrices of $512 \times 510$ pixels with $17 \mu \mathrm{m}$ pitch. They are stitched together along one direction accross the wafer. Ladders of 5 or 7 chips are being achieved in this way. They will offer the first opportunity to test on real scale the performances observed with small scale prototypes, to investigate the chip production yield and the properties of stitching, and to experience the read-out of millions of pixels.

Finally, a crucial issue of the next years will be the design of fast read-out micro-circuits including sparsification, integrated on the sensor substrate.

\section{Summary and Conclusion}

First prototypes of CMOS sensors designed for charged particle tracking show that this new detection technique provides excellent performances (e.g. $\mathrm{S} / \mathrm{N} \sim 40$, detection efficiency $>99 \%$, spatial resolution $\sim 1.5 \mu \mathrm{m}$ ). Whether the latter can be reproduced on real scale will come out from the tests of the first large device, currently fabricated.

The coming three years are expected to allow designing fast read-out micro-circuits with sparsification integrated on the chip as well as to explore extensively the real potential of this technology. The outcome of this programe will determine in which extent CMOS sensors can be used as building blocks of future vertex detectors.

\section{References}

[1] B.Dierickx, G.Meynants, D.Scheffer, Proceedings of the IEEE CCD AIS workshop, Brugge, Belgium, 5-7 June (1997), p.P1;

[2] R.Turchetta et al., Nucl.Instr. and Meth. A 458 (2001)677;

[3] M.Winter et al., Proceedings of IEEE NSS Conference, October 2000, Lyon, France;

[4] Y.Gornushkin et al., Proceedings of the Vienna Conference on Instrumentation, February 2001, Vienna, Austria, and references therein;

[5] G.Deptuch et al., Nucl.Instr. and Meth. A 465 (2001) 92. 